\title{
ALTAS CAPACIDADES Y COGNICIÓN SOCIAL
}

\author{
ALTAS CAPACIDADES E COGNIÇÃO SOCIAL \\ HIGH ABILITIES AND SOCIAL COGNITION
}

\author{
Ricardo QUINTERO RODRÍGUEZ ${ }^{1}$ \\ Triana AGUIRRE DELGADO ${ }^{2}$ \\ África BORGES DEL ROSAL ${ }^{3}$
}

RESUMEN: Las altas capacidades, multidimensionalmente configuradas, más allá de un CI elevado, son resultado tanto de un proceso de desarrollo madurativo como psicosocial precoz. Por consiguiente, un campo de estudio de especial interés es la cognición social, un campo que engloba a los procesos mentales que perciben, captan e interpretan la información social del entorno. El presente estudio busca determinar las diferencias existentes entre el alumnado con altas capacidades comparándolo con sus pares de inteligencia normativa. Atendiendo a ello, se realizó un muestreo intencional en adolescentes, obteniendo una muestra de 146 estudiantes de Enseñanza Secundaria Se utilizó un diseño transversal con metodología de encuesta. Los resultados avalan las hipótesis planteadas. No obstante, se debate la relevancia de estos aspectos diferenciales entre el alumnado de altas capacidades y sus pares.

PALABRAS CLAVE Altas capacidades. Cognición social. Adolescentes.

RESUMO: Altas capacidades, configuradas multidimensionalmente, além de um alto QI, são o resultado tanto de um processo precoce de maturação quanto de um processo de desenvolvimento psicossocial. Portanto, um campo de estudo de especial interesse é a cognição social, um campo que engloba os processos mentais que percebem, capturam e interpretam as informações sociais do meio ambiente. O presente estudo procura determinar as diferenças entre estudantes altamente capacitados e seus pares normativamente inteligentes. Com isto em mente, foi obtida uma amostra proposital de 146 alunos do Ensino Médio. Foi utilizado um projeto transversal com metodologia de pesquisa. Os resultados apóiam as hipóteses apresentadas. Entretanto, a relevância desses aspectos diferenciais entre os estudantes de alta capacidade e seus pares é discutida.

PALAVRAS-CHAVE: Altas habilidades. Cognição social. Adolescentes.

\footnotetext{
${ }^{1}$ Universidad de La Laguna (ULL), San Cristóbal de La Laguna - Canarias - España. Alumno de Posgrado, Departamento de Psicología Clínica, Psicobiología y Metodología. ORCID: https://orcid.org/0000-0002-51364504. E-mail: ricardo.quintero.15@ull.edu.es

${ }^{2}$ Universidad de La Laguna (ULL), San Cristóbal de La Laguna - Canarias - España. Personal Investigador en Formación, Departamento de Psicología Clínica, Psicobiología y Metodología. ORCID: https://orcid.org/00000002-2110-1551. E-mail: taguirre@ull.edu.es

${ }^{3}$ Universidad de La Laguna (ULL), San Cristóbal de La Laguna - Canarias - España. Catedrática de Universidad, Departamento de Psicología Clínica, Psicobiología y Metodología. ORCID: https://orcid.org/00000001-8267-4401. E-mail: aborges@ull.edu.es
} 
ABSTRACT: High abilities, multidimensionally configured, beyond a high IQ, are the result of both an early maturational and psychosocial developmental process. Therefore, a field of study of special interest is social cognition, a field that encompasses the mental processes that perceive, capture and interpret social information from the environment. The present study seeks to determine the differences between highly able students and their normatively intelligent peers. With this in mind, a purposive sample of 146 Secondary School students was obtained. A cross-sectional design with survey methodology was used. The results support the hypotheses put forward. However, the relevance of these differential aspects between high ability students and their peers is discussed.

KEYWORDS: High abilities. Social cognition. Adolescents.

\section{Introducción}

Durante siglos, la inteligencia ha sido motivo de numerosas investigaciones y controversias. Particularmente, las primeras reflexiones de la inteligencia y su naturaleza no fueron realizadas por educadores ni psicólogos, sino fundamentadas desde el ámbito filosófico. No obstante, desde estas primeras aproximaciones filosóficas, la literatura especializada ha generado distintos enfoques teóricos y definiciones de la inteligencia, conceptualizándola como: (a) una concepción de una inteligencia monolítica que describe la inteligencia como un componente unitario; (b) un enfoque factorial que la describe a partir de una serie de componentes o actitudes; o (c) una estructuración jerárquica que sustenta un enfoque integrador a partir de los planteamientos anteriores (LÓPEZ, 2013).

De manera análoga, se ha evidenciado una proliferación de definiciones utilizadas para describir conceptualmente a la inteligencia y, por ende, el fenómeno de las altas capacidades (AACC). Por un lado, se plantean enfoques que sostienen que las AACC vienen determinadas por un rasgo interno, es decir, un rasgo medible, real o concreto, basándose en un enfoque psicométrico del término (ROEPER, 1982). Ahora bien, otros enfoques afirman que las AACC no reflejarían un hecho natural, sino una construcción social, generada para categorizar a una población que posee un desempeño excepcional o en su defecto, rinden de manera sobresaliente en cualquiera de los aspectos que la sociedad o cultura considera relevantes (BORLAND, 2009; PFEIFFER, 2017).

En efecto, las AACC al ser un constructo que se encuentra ligado intrínsecamente a la inteligencia, evoluciona a la par que la evidencia empírica en su conceptualización (BORGES; HERNÁNDEZ-JORGE, 2006; BORGES; HERNÁNDEZ-JORGE; RODRÍGUEZ-NAVEIRAS, 2016; COMES et al., 2008). Por ello, se ha evidenciado un cambio en la concepción de las AACC, desde una concepción estática, que relacionaba al 
individuo como aquel que obtenía una puntuación relativamente superior a sus iguales en una prueba de inteligencia, a una concepción del término como un conjunto de aptitudes configuradas de manera dinámica, desarrolladas a lo largo de la vida e influenciadas por el entorno. En resumen, las AACC constituyen la función derivada de un proceso de desarrollo, conformado según las variables psicosociales incidentes en él, la educación y el sustrato neurobiológico subyacente (OLSZEWSKI-KUBILIUS; SUBOTNIK; WORRELL, 2015; PFEIFFER, 2015; SASTRE-RIBA, 2014)

Un área de investigación de gran interés en AACC es la que estudia las diferencias del colectivo con muestras comunitarias, tanto en aspectos cognitivos (RODRÍGUEZNAVEIRAS et al., 2019) como socioafectivos (HERNÁNDEZ-LASTIRI; BORGES; CADENAS, 2019; VALADEZ et al., 2020). En este sentido, se presenta al colectivo de las AACC como una población de especial interés para el estudio de la cognición social (CS), dadas sus características diferenciales respecto a sus pares de inteligencia normativa: Un ritmo de aprendizaje más rápido, que implicaría una temprana comprensión y resolución de problemas, un mayor uso de estrategias y capacidad de adaptación social que promueven la resolución de problemas complejos de forma eficiente, una mayor autorregulación y flexibilidad cognitiva que le permiten promover un alto compromiso y/o motivación hacia sus objetivos, e incluso, una mayor resistencia a la interferencia ofrecidas por el entorno, que les permitiría ser productores creativos y competentes de alternativas. No obstante, estas características suelen ir ligadas al concepto de asincronía, dando lugar a un desajuste entre el desarrollo cognitivo y el socio-emocional (PFEIFFER, 2017; RENZULLI, 1990; SASTRERIBA, 2014; VALADEZ; BETANCOURT; ZAVALA, 2006).

Acorde a lo que se ha expuesto, se describe a la CS como un campo de estudio que permite englobar los procesos mentales (psicológicos, neuropsicológicos y sociales) que perciben, captan e interpretan la información social del entorno dando lugar a una respuesta en base al contexto, lo que permitiría poseer estructuras sociales complejas (ADOLPHS, 1999; 2001). Aunque la propuesta de este autor es compartida y aprobada, se trata de un concepto que ha generado controversias y diversas teorías que han diferenciado o integrado planteamientos de corte social y cognitivo (BEER; OCHSNER, 2006; CONDOR; ANTAKI, 2000; HUMFRESS et al., 2002). Asimismo, se ha establecido una división de la CS en cuatro dominios diferenciados: (a) entendimiento de los otros, que incluye Teoría de la Mente (ToM) y empatía, caracterizados por permitir la comprensión de los estados mentales internos y externos; (b) la autocomprensión o comprensión de uno mismo; (c) El autocontrol, proceso 
que permite la regulación y revalorización de los impulsos; (d) la interacción producida entre la interfaz del mundo interno y el externo (LIEBERMAN, 2007).

El primer dominio presentaría especial importancia para la comprensión de los otros, la empatía y la ToM. En este sentido, los primeros estudios que abordaron la conceptualización de la ToM fueron realizados en animales y permitieron caracterizarla como la capacidad para predecir el comportamiento de otros a partir de la atribución de estados mentales (PREMACK; WOODRUFF, 1978). Posteriormente, se iniciaron los estudios en seres humanos, desarrollándose una prueba que permitió la compresión de este estado mental denominado "falsa creencia", la prueba de Sally y Anne (WIMMER, 1983). Por otra parte, las primeras investigaciones que abordarían la conceptualización de la empatía presentaban enfoques opuestos, constituyendo una perspectiva cognitiva y otra afectiva. Desde la primera, se comprendía la empatía como un proceso que implica la comprensión de la situación desde el punto de vista de la otra persona (HOGAN, 1969). En la segunda implicaría la capacidad de sentir lo que la otra persona percibe en términos emocionales (STOTLAND, 1969). Posteriormente, Davis (1983) propone un modelo integrador y multidimensional, conceptualizándola como una habilidad que permite identificar los estados mentales del resto $\mathrm{y}$ diferenciarlos de los propios, recogiendo una definición englobando aspectos afectivos y cognitivos.

El segundo dominio, propuesto por Lieberman (2007), presta especial atención a la comprensión de uno mismo, un conjunto de habilidades que permiten representarnos a nosotros mismos, regular nuestro comportamiento y diferenciarnos del resto. En esta línea, el bienestar y la salud mental cobran relevancia en el desarrollo del funcionamiento mental idóneo. Históricamente, la conceptualización del término bienestar ha estado supeditado a diversos conceptos (v.g. autoconocimiento, felicidad, calidad de vida, salud mental) (GARCÍA-ALANDETE, 2014; VÁZQUEZ et al., 2009). En esta línea, este término ha dado lugar a dos corrientes conceptuales: (a) una perspectiva hedónica o vertiente emocional que engloba estados de ánimo placenteros y de felicidad, relacionándolo con el denominado bienestar emocional; y (b) una perspectiva eudamónica, que comprende la vertiente cognitiva del concepto, abarcando la consecución de metas vitales y el deseo de sentirse realizado, relacionándose con el bienestar psicológico (RYAN; DECI, 2001). En consonancia, diversos autores han integrado conceptos como salud mental y bienestar, considerándolos, junto a otras variables sociales, como predictores del correcto desempeño en el ámbito social (GALDERISI et al., 2015; VÁZQUEZ et al., 2009). 
Por último, desde el modelo propuesto por Lieberman (2007) otro aspecto que constituye el constructo de la CS correspondería a la interacción producida entre el mundo interno y el externo, es decir, la competencia social, conformada por las habilidades sociales (HHSS), descritas como conductas adaptativas y/o de solución de conflictos que permiten la adecuación al entorno social, ya sean con un carácter básico, avanzado o instrumental (GOLDSTEIN, 1980). El aprendizaje de dichas habilidades viene determinado por partir un enorme carácter aprendido, mediatizado por un proceso de interacción social (BANDURA; MCCLELLAND, 1977). Asimismo, se han planteado numerosos estudios que evidencian relaciones entre las HHSS y la ToM, estableciendo interacciones recíprocas entre sus respectivos componentes (ADOLPHS, 2001; AGUILAR et al., 2016; DECETY, 2010; LIEBERMAN, 2007).

En concordancia a lo planteado inicialmente, se presentan posturas antagónicas y contradictorias en lo que respecta al estado de ajuste emocional y psicosocial de las personas con AACC (MARTIN; BURNS; SCHONLAU, 2010). Por un lado, existen investigaciones que plantean desde una hipótesis armónica en las AACC donde se ha evidenciado un igual o mayor ajuste psicológico, funcionamiento mentalista, HHSS y empatía en estos individuos frente a sus pares de inteligencia normativa (BORGES; HERNÁNDEZ-JORGE; RODRÍGUEZ-NAVEIRAS, 2011; LEIVA, 2007; LÓPEZ; SOTILLO, 2009; RODRÍGUEZNAVEIRAS et al., 2019). Por otro lado, otras investigaciones concluyen que las personas con AACC presentan incremento de la vulnerabilidad de los problemas de ajuste emocional, lo que produciría cambios en el funcionamiento psicológico normal de dicha población en materia de bienestar y/o salud mental (BAILEY, 2011; RAMIRO et al., 2016).

Por consiguiente, el objetivo de la presente investigación es analizar las diferencias existentes para las variables influenciadas por el constructo de la CS, estableciendo una comparación entre el alumnado con AACC y sus pares de muestra comunitaria.

En este sentido, considerando que la naturaleza de la CS está mayoritariamente influenciada por aspectos cognitivos, destacables en el colectivo con AACC, se plantea como hipótesis que un desempeño de este alumnado mayor o igual que el de sus iguales con inteligencia normativa, en las dimensiones que intervienen la CS. 


\section{Método}

\section{Diseño}

El presente se realizó mediante metodología de encuestas, diseño transversal.

\section{Participantes}

La muestra estudiada está compuesta por 146 adolescentes, de los cuales 65 eran mujeres, con edades comprendidas entre los 12 y 18 años, pertenecientes a siete centros educativos y una entidad privada especializada en el desarrollo integral de adolescentes con AACC, en la comunidad autónoma de Canarias.

La selección de la submuestra objeto del presente estudio se realizó mediante un muestreo intencional, utilizando la puntuación de los tests de inteligencia empleado en la investigación (CATTELL; CATTELL; WEISS, 2017; HERRANZ, 2017), obteniéndose así dos grupos: (a) el primero, que conforma a la muestra comunitaria, constituido por los participantes que obtuvieron puntuaciones correspondientes a los percentiles 45 y 55 en alguno de los tests, en cada nivel educativo; y (b) el grupo, de alumnado de altas capacidades, con los participantes que obtuvieron puntuaciones superiores al percentil 90, en cada nivel educativo, además, con los participantes con un diagnóstico positivo. La distribución de los participantes por curso y grupo se presenta en la Tabla 1.

Tabla 1 - Estadísticos descriptivos de los/as participantes de la muestra general

\begin{tabular}{ccccccc}
\hline Curso & \multicolumn{1}{c}{$\mathrm{N}$} & \multicolumn{2}{c}{ Edad } & \multicolumn{2}{c}{ DT } \\
\hline Grupo & Muestra comunitaria & AACC & $\begin{array}{c}\text { Muestra } \\
\text { comunitaria }\end{array}$ & AACC & $\begin{array}{c}\text { Muestra } \\
\text { comunitaria }\end{array}$ & AACC \\
\hline $1^{\circ}$ ESO & 14 & 22 & 13,00 & 12,74 & 0,604 & 0,453 \\
$2^{\circ} \mathrm{ESO}$ & 8 & 12 & 13,87 & 13,60 & 0,589 & 0,418 \\
$3^{\circ} \mathrm{ESO}$ & 32 & 22 & 14,83 & 14,51 & 0,448 & 0,754 \\
$4^{\circ} \mathrm{ESO}$ & 19 & 17 & 16,00 & 15,71 & 1,023 & 0,518 \\
Total & 73 & 73 & 14,68 & 14,10 & 1,243 & 1,259 \\
\hline
\end{tabular}

Fuente: Elaboración propia

\section{Instrumentos}

Las pruebas utilizadas se describen a continuación. Todas ellas, se encuentran baremadas al castellano y cuentan autorización de uso de los autores. De igual manera, se presenta la fiabilidad calculada para la muestra (véase Tabla 2). 
Tabla 2 - Fiabilidades de las pruebas y sus escalas, calculadas para la muestra

\begin{tabular}{|c|c|c|c|}
\hline Prueba & $\alpha$ & Escalas & $\alpha$ \\
\hline Health Questionnaire & 0,896 & - & - \\
\hline Factor G de Herranz & 0,680 & - & - \\
\hline \multirow[t]{6}{*}{ Kidscreen-27 } & 0,872 & & \\
\hline & & Bienestar físico & 0,784 \\
\hline & & Bienestar Psicológico & 0,520 \\
\hline & & Autonomía y relación con los padres & 0,794 \\
\hline & & Apoyo social y pares & 0,828 \\
\hline & & Entorno escolar & 0,762 \\
\hline \multirow[t]{5}{*}{ Interpersonal Reactivity Index } & 0,788 & & \\
\hline & & Toma de perspectiva & 0,709 \\
\hline & & Fantasía & 0,692 \\
\hline & & Preocupación empática & 0,666 \\
\hline & & Malestar personal & 0,709 \\
\hline \multirow[t]{7}{*}{ Habilidades Sociales de Goldstein } & 0,937 & & \\
\hline & & Primeras habilidades sociales & 0,677 \\
\hline & & Habilidades sociales avanzadas & 0,514 \\
\hline & & Habilidades relacionadas con los sentimientos & 0,738 \\
\hline & & Habilidades alternativas a la agresión & 0,776 \\
\hline & & Habilidades para hacer frente al estrés & 0,810 \\
\hline & & Habilidades de planificación & 0,785 \\
\hline \multirow[t]{4}{*}{ Historias Extrañas de Happé } & 0,782 & & \\
\hline & & Historias mentales & 0,379 \\
\hline & & Historias físicas & 0,637 \\
\hline & & Historias desvinculadas & 0,814 \\
\hline \multicolumn{4}{|c|}{$\begin{array}{l}\text { Nota. No fue posible calcular la fiabilidad de la prueba de inteligencia: Factor g-R. Test de Inteligencia No } \\
\text { Verbal - Revisado. La prueba solo indica la puntuación total obtenida por escala y la prueba. En este estudio, las } \\
\text { pruebas de inteligencia se han utilizado como criterio de categorización de los adolescentes para los grupos de } \\
\text { estudio, junto a los diagnósticos positivos de AACC. }\end{array}$} \\
\hline
\end{tabular}

Fuente: Elaboración propia

Factor $G$ de Herranz, desarrollado como una versión reducida del Test Informatizado para la Medida de la Inteligencia General (TIMIG) de Herranz (2017). La prueba mide el factor general de inteligencia. Esta prueba, generada a partir de la teoría de respuesta al ítem (TRI), se configura mediante una escala continua que utiliza preguntas de elección múltiple con cuatro alternativas de respuesta. Atendiendo a ello, en la presente investigación, se adaptó dicha escala atendiendo al nivel de dificultad. No obstante, de los 169 ítems que la conforman, se utilizaron 40. El estudio original reporta un alfa de Cronbach situado entre 0,684 y 0,912, según las subescalas utilizadas.

Factor g-R Test de Inteligencia No Verbal - Revisado (CATTELL; CATTELL; WEISS, 2017). La prueba mide el factor general de inteligencia en una escala continua que utiliza preguntas de elección múltiple con cinco alternativas de respuesta. Esta prueba presenta una consistencia interna situada entre 0,74 y 0,83 , en su baremación para estudiantes de secundaria. 
Health Questionnaire (GHQ-12), elaborado por Goldberg (1988); adaptación española de Sánchez-López y Dresch (2008). Esta prueba indaga sobre la presencia de síntomas emocionales (depresión y ansiedad). Se utiliza como screening para detectar trastornos mentales recientes. La prueba presenta 12 ítems y utiliza una escala tipo Likert de 0 a 3 . Una puntuación más alta implicaría un mayor grado de sintomatología emocional. Una puntuación de 12 o superior indicaría la existencia de un posible trastorno emocional. Esta prueba presenta una elevada consistencia interna, su valor es 0,86 .

Kidscreen-27, desarrollado como una versión reducida del Kidscreen-52 de RavensSieberer et al. (2008). La prueba presenta 27 ítems, que se distribuyen en cinco dimensiones: (a) Bienestar físico; (b) Bienestar psicológico; (c) Autonomía y padres; (d) Amigos y apoyo social; (e) Entorno escolar. La prueba utiliza una escala tipo Likert de 1 a 5 . Además, proporciona un perfil de salud, a través de la interpretación de sus respectivas dimensiones. La prueba presenta una elevada consistencia interna, situándose entre el 0,80 y 0,84 .

Interpersonal Reactivity Index, elaborado por Davis (1980); adaptación española de Mestre et al. (2004). Este instrumento evalúa el concepto global de empatía desde una perspectiva multidimensional que recoge dos factores emocionales y dos factores cognitivos. Estas dimensiones cognitivas y emocionales, respectivamente, son: (a) Toma de perspectiva; (b) Fantasía; (c) Preocupación empática; (d) Malestar personal. La prueba mantiene una escala tipo Likert de 1 a 5, con 28 ítems. La prueba presenta una consistencia interna que oscila entre el 0,56 y 0,70 .

Escala de Habilidades Sociales (Goldstein et al., 1980; adaptación al español por Tomas (1995). La prueba está compuesta por 50 ítems, representados en seis grupos: (a) Primeras Habilidades Básicas; (b) Habilidades Sociales Avanzadas; (c) Habilidades relacionadas con los Sentimientos; (d) Habilidades Alternativas a la Agresión; (e) Habilidades para hacer frente al Estrés; (f) Habilidades de Planificación. Se compone de una escala tipo Likert de 1 a 5 . La prueba presenta una elevada consistencia interna, su valor es 0,92.

Historias Extrañas de Happé (Happé, 1994; adaptación española por Aguilar et al. 2014). La prueba está compuesta por 24 historias, diseñadas para evaluar las habilidades mentalistas avanzadas, a través de la intencionalidad de expresiones comunicativas no explicitas. Las historias pueden dividirse en tres factores: Historias Físicas, Historias Mentales e Historias Desvinculadas. Las respuestas se codifican en una escala tipo Likert de 0 a 2. La prueba presenta una consistencia interna de 0,78 . Se muestra la fiabilidad de la codificación realizada para la muestra (véase Tabla 3). 
Tabla 3 - Fiabilidad inter-jueces de la prueba de Historias Extrañas de Happé

\begin{tabular}{ccccccccc}
\hline Ítem & $\kappa$ & Ítem & $\kappa$ & Ítem & $\kappa$ & Ítem & $\kappa$ & Ítem \\
\hline 1 & 0,896 & 7 & 0,964 & 13 & 0,963 & 19 & 1 & 13 \\
2 & 0,872 & 8 & 0,821 & 14 & 1 & 20 & 0,901 & 14 \\
3 & 0,932 & 9 & 0,966 & 15 & 0,956 & 21 & 1 & 15 \\
4 & 0,875 & 10 & 0,891 & 16 & 0,904 & 22 & 1 & 16 \\
5 & 0,939 & 11 & 1 & 17 & 1 & 23 & 0,847 & 17 \\
6 & 0,876 & 12 & 0,924 & 18 & 0,892 & 24 & 1 & 18 \\
\hline
\end{tabular}

Nota. Todos los ítems de la prueba fueron corregidos por dos jueces. La fiabilidad inter-jueces fue realizada para garantizar la correcta categorización de las respuestas dadas en la prueba de Historias Extrañas de Happé, asegurando la estabilidad de los criterios de clasificación planteados por sus autores. Se calculó la fiabilidad a partir del coeficiente Kappa de Cohen $(\kappa)$.

Fuente: Elaboración propia

\section{Procedimiento}

En primer lugar, esta investigación fue aprobada por el Comité de Ética de la Investigación y Bienestar Animal de la Universidad de La Laguna (registro CEIBA20210441).

Posteriormente, se contactó con las entidades, a las que se solicitó autorización, así como a los progenitores y/o tutores legales, quienes firmaron consentimiento informado. De esta manera, se garantizó el carácter voluntario y la conservación del anonimato cumpliendo con la Ley Orgánica 3/2018, de 5 de diciembre, de Protección de Datos Personales y garantía de los derechos digitales.

La administración de los cuestionarios se realizó en los centros educativos o entidades, de forma colectiva y en presencia de los evaluadores. Los instrumentos utilizados fueron cumplimentados en formato informatizado a través de Google Formularios. Se presenta el protocolo de administración de las pruebas (véase Tabla 4).

Tabla 4 - Protocolo de administración de las pruebas

\begin{tabular}{ccc}
\hline Instrumento & Orden & Duración \\
\hline General Health Questionnaire (GHQ-12) & $1^{\text {o }}$ & $5-10$ \\
Factor G de Herranz & $2^{\text {o }}$ & $30-40$ \\
Kidscreen-27 & $3^{\text {o }}$ & $10-15$ \\
Interpersonal Reactivity Index (IRI) & $4^{\text {o }}$ & 10 \\
Habilidades Sociales de Goldstein & $5^{\text {o }}$ & 10 \\
Historias Extrañas de Happé & $6^{\text {o }}$ & $30-40$ \\
\hline
\end{tabular}

Nota. Se utilizó como criterio de categorización, una de las dos pruebas de inteligencia utilizadas en el presente estudio: (a) Factor G de Herranz; (b) Factor g-R Test de Inteligencia No Verbal - Revisado. Las pruebas se administraron, siempre, en segundo lugar.

Fuente: Elaboración propia 


\section{Análisis de datos}

Para contrastar la hipótesis de ausencia de diferencias en las variables de cognición social estudiadas, se ha procedido a realizar análisis multivariados de la varianza (MANOVA), así como una prueba t de Student para muestras independientes, que permitirán determinar la influencia de la inteligencia en las variables de estudio. Los análisis se han realizado con el programa SPSS, en su versión de prueba y el programa Jamovi, en su versión 1.6.7.

\section{Resultados}

\section{Salud mental}

Para contrastar si existen diferencias en salud mental entre los dos grupos se aplicó una prueba $\mathrm{t}$ de Student de la prueba GHQ-12 (GOLDBERG; WILLIAMS, 1988; SÁNCHEZ-LÓPEZ; DRESCH, 2008). Los estadísticos descriptivos se pueden observar en la Tabla 5.

Tabla 5 - Descriptivos GHQ-12 por grupo

\begin{tabular}{cccc} 
Grupo & N & Media & DT \\
\hline Muestra comunitaria & 73 & 13,01 & 7,056 \\
AACC & 73 & 12,25 & 6,964 \\
\hline
\end{tabular}

Fuente: Elaboración propia

No se obtuvieron diferencias significativas entre ambos grupos $(\mathrm{t}=0,661 \mathrm{p}>0,05)$, resultando un tamaño de efecto muy pequeño $(\mathrm{d}=0,108)$. No obstante, ambos grupos superan el punto de corte que señalan los autores de la prueba de una adecuada salud mental (puntuación de 12 o inferior), indicando la existencia de un posible trastorno emocional.

\section{Bienestar}

Para determinar si hay diferencias en bienestar entre ambos grupos de estudiantes se realizó un análisis MANOVA en relación con las cinco dimensiones de bienestar propuestas en la prueba Kidscreen-27 (RAVENS-SIEBERER et al., 2008). Los estadísticos descriptivos se muestran en la Tabla 6. 
Tabla 6 - Estadísticos descriptivos del Kidscreen-27 por grupo

\begin{tabular}{llllll}
\hline \multicolumn{1}{c}{ Escala } & \multicolumn{1}{c}{ Grupo } & $\mathrm{N}$ & Media & DT \\
\hline \multirow{2}{*}{ Bienestar físico } & Muestra comunitaria & 73 & & 16,26 & 3,701 \\
& AACC & 73 & & 16,34 & 3,667 \\
Bienestar Psicológico & Muestra comunitaria & 73 & 23,38 & 3,247 \\
& AACC & 73 & 22,64 & 3,970 \\
\multirow{2}{*}{ Autonomía y relación con los padres } & Muestra comunitaria & 73 & 26,97 & 5,310 \\
& AACC & 73 & 27,79 & 4,708 \\
Apoyo social y pares & Muestra comunitaria & 73 & 16,93 & 2,810 \\
\multirow{2}{*}{ Entorno escolar } & AACC & 73 & 15,03 & 3,559 \\
& Muestra comunitaria & 73 & 14,36 & 2,810 \\
& AACC & 73 & 14,74 & 3,028 \\
\hline
\end{tabular}

Fuente: Elaboración propia

Se comprobó el cumplimento del supuesto de igualdad de matrices de varianzacovarianza, aceptándose la hipótesis nula de que no existen diferencias entre las matrices de covarianzas observadas de las variables dependientes entre los grupos (véase Tabla 7).

Tabla 7 - Prueba de Box de la igualdad de matrices de covarianzas

\begin{tabular}{lcccccc}
\hline & Prueba & M de Box & F & gl1 & gl2 & Sig. \\
\hline Kidscreen-27 & & 16,29 & 1,046 & 15 & 83489,684 & 0,403 \\
\hline
\end{tabular}

Fuente: Elaboración propia

Se comprobó la homocedasticidad con el estadístico de Levene, cumpliéndose el supuesto en todas las escalas (véase Tabla 8).

Tabla 8 - Homocedasticidad Kidscreen-27

\begin{tabular}{lcccc}
\hline \multicolumn{1}{c}{ Escala } & Estadístico de Levene & gl1 & g12 & Sig. \\
\hline Bienestar físico & 0,263 & 1 & 144 & 0,609 \\
Bienestar Psicológico & 1,662 & 1 & 144 & 0,199 \\
Autonomía y relación con los padres & 0,520 & 1 & 144 & 0,472 \\
Apoyo social y pares & 2,200 & 1 & 144 & 0,140 \\
Entorno escolar & 0,000 & 1 & 144 & 0,991 \\
\hline
\end{tabular}

\section{Fuente: Elaboración propia}

Asimismo, el contraste resultó significativo ( $\lambda$ de Wilks $=0,868, \mathrm{~F}_{(5,140)}=4,251$, $\mathrm{p}=0,001, \eta^{2}$ parcial $\left.=0,132, \beta=0,957\right)$. No obstante, los resultados individuales del ANOVA muestran únicamente diferencias significativas en la escala de Apoyo Social y Pares, con un tamaño de efecto mediano. En la tabla 9 se muestra las pruebas inter-sujeto para el Kidscreen27. 
Tabla 9 - Pruebas de efectos inter-sujetos en Kidscreen-27

\begin{tabular}{ccccc}
\hline Escala & $\mathrm{F}_{1,146}$ & Sig. & $\eta^{2}$ parcial & $\beta$ \\
\hline Bienestar físico & 0,018 & 0,893 & 0,000 & 0,052 \\
Bienestar Psicológico & 1,519 & 0,220 & 0,010 & 0,232 \\
Autonomía y relación con los padres & 0,979 & 0,324 & 0,007 & 0,166 \\
Apoyo social y pares & 12,853 & 0,000 & 0,082 & 0,945 \\
Entorno escolar & 0,629 & 0,429 & 0,004 & 0,124 \\
\hline
\end{tabular}

Fuente: Elaboración propia

\section{Empatía}

Con el objetivo de estudiar si existían diferencias significativas para ambos grupos en relación en las dimensiones de la prueba Interpersonal Reactivity Index(DAVIS, 1980) se realizó un MANOVA. En la tabla 10 se presentan los estadísticos descriptivos.

Tabla 10 - Estadísticos descriptivos del Interpersonal Reactivity Index por grupo

\begin{tabular}{llccc}
\hline \multicolumn{1}{c}{ Escala } & \multicolumn{1}{c}{ Grupo } & N & Media & DT \\
\hline \multirow{2}{*}{ Toma de perspectiva } & Muestra comunitaria & 73 & 16,01 & 4,486 \\
\multirow{3}{*}{ Fantasía } & AACC & 73 & 15,93 & 5,796 \\
\multirow{2}{*}{ Preocupación empática } & Muestra comunitaria & 73 & 14,78 & 5,546 \\
\multirow{2}{*}{ Malestar personal } & AACC & 73 & 14,64 & 5,616 \\
& Muestra comunitaria & 73 & 17,08 & 4,521 \\
& AACC & 73 & 17,75 & 4,612 \\
& Muestra comunitaria & 73 & 10,48 & 4,429 \\
& AACC & 73 & 11,64 & 5,373 \\
\hline
\end{tabular}

Fuente: Elaboración propia

Se comprueba que se cumple el supuesto de igualdad de matrices de varianzacovarianza (véase Tabla 11).

Tabla 11 - Prueba de Box de la igualdad de matrices de covarianzas

\begin{tabular}{cccccc}
\hline Prueba & M de Box & $\mathrm{F}$ & $\mathrm{gl}^{1}$ & $\mathrm{gl}^{2}$ & Sig. \\
\hline Interpersonal Reactivity Index & 13,949 & 1,353 & 10 & 99136,255 & 0,196 \\
\hline
\end{tabular}

Fuente: Elaboración propia

Sin embargo, el contraste no resultó significativo ( $\lambda$ de Wilks $=0,983, F_{(4,141)}=0,606$, $\mathrm{p}=0,659, \mathrm{n}^{2}$ parcial $\left.=0,017\right)$. 


\section{Habilidades sociales}

Para determinar si existían diferencias significativas en las diferentes dimensiones propuestas desde la Escala de Habilidades Sociales (GOLDSTEIN, 1980 ; TOMAS, 1995) se realizó un MANOVA. Los estadísticos descriptivos se exponen en la Tabla 12.

Tabla 12 - Estadísticos descriptivos de la Escala de Habilidades Sociales por grupo

\begin{tabular}{ccccc}
\hline Escala & Grupo & $\mathrm{N}$ & Media & DT \\
\hline Primeras habilidades & Muestra comunitaria & 73 & 24,07 & 3,928 \\
sociales & AACC & 73 & 22,27 & 3,497 \\
Habilidades sociales & Muestra comunitaria & 73 & 16,81 & 2,701 \\
avanzadas & AACC & 73 & 17,11 & 2,923 \\
Habilidades relacionadas & Muestra comunitaria & 73 & 20,09 & 3,852 \\
con los sentimientos & AACC & 73 & 18,85 & 3,665 \\
Habilidades alternativas & Muestra comunitaria & 73 & 25,71 & 4,287 \\
a la agresión & AACC & 73 & 26,16 & 4,899 \\
Habilidades para hacer & Muestra comunitaria & 73 & 33,36 & 5,987 \\
frente al estrés & AACC & 73 & 32,85 & 5,737 \\
Habilidades de & Muestra comunitaria & 73 & 23,51 & 4,321 \\
planificación & AACC & 73 & 23,05 & 4,027 \\
\hline
\end{tabular}

Fuente: Elaboración propia

Asimismo, se realiza la Prueba de Box de la igualdad de matrices de covarianzas y se concluye el cumplimento del supuesto de igualdad (véase Tabla 13).

Tabla 13 - Prueba de Box de la igualdad de matrices de covarianzas

\begin{tabular}{cccccc}
\hline Prueba & M de Box & F & gl1 & gl2 & Sig. \\
\hline Habilidades Sociales de Goldstein & 21,020 & 0,956 & 21 & 76266,974 & 0,516 \\
\hline
\end{tabular}

Fuente: Elaboración propia

La homocedasticidad fue calculada con el estadístico de Levene, resultando todas las escalas homocedásticas (véase Tabla 14).

Tabla 14 - Homocedasticidad Habilidades Sociales de Goldstein

\begin{tabular}{lcccc}
\hline \multicolumn{1}{c}{ Escala } & Estadístico de Levene & gl1 & gl2 & Sig. \\
\hline Primeras habilidades sociales & 1,093 & 1 & 144 & 0,298 \\
Habilidades sociales avanzadas & 1,086 & 1 & 144 & 0,299 \\
Habilidades relacionadas con los sentimientos & 0,349 & 1 & 144 & 0,556 \\
Habilidades alternativas a la agresión & 0,529 & 1 & 144 & 0,468 \\
Habilidades para hacer frente al estrés & 0,099 & 1 & 144 & 0,753 \\
Habilidades de planificación & 0,623 & 1 & 144 & 0,431
\end{tabular}

Fuente: Elaboración propia 
El contraste resultó significativo $\left(\lambda\right.$ de Wilks $=0,855, F_{(6,139)}=3,928, p=0,001, \eta^{2}$ parcial $=0,145, \beta=0,965)$.

Los resultados individuales del ANOVA muestran diferencias significativas en las dimensiones "Primeras habilidades sociales" y "Habilidades relacionadas con los sentimientos", con un tamaño de efecto medio y bajo, respectivamente. Se presentan las pruebas de efectos inter-sujeto para la Escala de Habilidades Sociales de Goldstein (véase Tabla 15).

Tabla 15 - Pruebas de efectos inter-sujetos en Habilidades Sociales de Goldstein

\begin{tabular}{|c|c|c|c|c|}
\hline Escala & $F_{1,146}$ & Sig. & $\eta^{2}$ parcial & $B$ \\
\hline Primeras habilidades sociales & 117,541 & 0,004 & 0,056 & 0,825 \\
\hline Habilidades sociales avanzadas & 3,315 & 0,519 & 0,003 & 0,098 \\
\hline Habilidades relacionadas con los sentimientos & 56,719 & 0,047 & 0,027 & 0,512 \\
\hline Habilidades alternativas a la agresión & 7,459 & 0,554 & 0,002 & 0,091 \\
\hline Habilidades para hacer frente al estrés & 9,377 & 0,602 & 0,002 & 0,081 \\
\hline Habilidades de planificación & 7,459 & 0,514 & 0,003 & 0,100 \\
\hline
\end{tabular}

Fuente: Elaboración propia

\section{Teoría de la mente}

Para determinar si existen diferencias significativas en las dimensiones propuestas por la prueba de Historias Extrañas de Happé (AGUILAR et al., 2014; HAPPÉ, 1994), se realizó un MANOVA. Los estadísticos descriptivos se exponen en la Tabla 16.

Tabla 16 - Estadísticos descriptivos de Historias extrañas de Happé por grupo

\begin{tabular}{lllcc}
\hline \multicolumn{1}{c}{ Escala } & \multicolumn{1}{c}{ Grupo } & $\mathrm{N}$ & Media & DT \\
\hline \multirow{2}{*}{ Historias mentales } & Muestra comunitaria & 73 & 9,93 & 2,244 \\
& AACC & 73 & 11,79 & 2,007 \\
\hline \multirow{2}{*}{ Historias físicas } & Muestra comunitaria & 73 & 10,23 & 3,116 \\
& AACC & 73 & 11,56 & 2,838 \\
\hline \multirow{2}{*}{ Historias desvinculadas } & Muestra comunitaria & 73 & 14,04 & 3,327 \\
& AACC & 73 & 14,82 & 2,653 \\
\hline
\end{tabular}

Fuente: Elaboración propia

Asimismo, se acepta el cumplimento del supuesto de igualdad de matrices de varianza-covarianza (véase Tabla 17). 
Tabla 17 - Prueba de Box de la igualdad de matrices de covarianzas

\begin{tabular}{cccccc}
\hline Prueba & M de Box & F & gl1 & gl2 & Sig. \\
\hline Historias extrañas de Happé & 6,651 & 1,083 & 6 & 150239,189 & 0,370 \\
\hline
\end{tabular}

Fuente: Elaboración propia

La homocedasticidad fue calculada con el estadístico de Levene, resultando todas las escalas homocedásticas (véase Tabla 18).

Tabla 18 - Homocedasticidad Historias extrañas de Happé por grupo

\begin{tabular}{lcccc}
\hline \multicolumn{1}{c}{ Escala } & Estadístico de Levene & gl1 & g12 & Sig. \\
\hline Historias mentales & 0,028 & 1 & 144 & 0,867 \\
Historias físicas & 0,375 & 1 & 144 & 0,541 \\
Historias desvinculadas & 3,339 & 1 & 144 & 0,070 \\
\hline
\end{tabular}

El contraste resultó significativo $\left(\lambda\right.$ de Wilks $=0,832, \mathrm{~F}_{(3,142)}=9,582, \mathrm{p}<0,001, \eta^{2}$ parcial $=0,168, \beta=0,997)$.

Los resultados individuales del ANOVA muestran diferencias significativas en el factor de "Historias mentales" e "Historias físicas", con un tamaño de efecto grande y bajo, respectivamente (véase Tabla 19).

Tabla 19 - Pruebas de efectos inter-sujeto en Historias extrañas de Happé

\begin{tabular}{lcccc}
\hline Escala & $\mathrm{F}_{1,146}$ & Sig. & $\eta^{2}$ parcial & $\beta$ \\
\hline Historias mentales & 27,955 & 0,000 & 0,163 & 1,000 \\
Historias físicas & 7,256 & 0,008 & 0,048 & 0,763 \\
Historias desvinculadas & 2,458 & 0,119 & 0,017 & 0,344 \\
\hline
\end{tabular}

\section{Discusión}

Las altas capacidades, multidimensionalmente configuradas, más allá de un CI elevado, son resultado tanto de un proceso de desarrollo madurativo como psicosocial precoz. Dicha conceptualización ha dado lugar a un interés creciente en estudiar las posibles diferencias de este colectivo con muestras comunitarias, tomando en consideración tanto los aspectos cognitivos (LEIVA, 2007; RODRÍGUEZ-NAVEIRAS et al., 2019; SASTRE-RIBA, 2008) como los socioafectivos (HERNÁNDEZ-LASTIRI; BORGES; CADENAS, 2019; VALADEZ et al., 2020).

De acuerdo con lo propuesto anteriormente, dado el carácter eminentemente cognitivo que viene mediatizado desde el constructo de la CS, el objetivo de la presente investigación ha sido analizar las diferencias existentes para las variables influenciadas por el constructo de 
la CS, estableciendo una comparación entre el alumnado con AACC y sus pares de inteligencia normativa.

La cognición social, a pesar de ser un campo ampliamente estudiado, con un amplio abanico de teorías explicativas (ADOLPHS, 1999; 2001; BEER; OCHSNER, 2006; CONDOR; ANTAKI, 2000; HUMFRESS et al., 2002; LIEBERMAN, 2007), se constituye como un constructo muy amplio, que conlleva tomar en consideración diversos factores y procesos en su estudio. Un primer aspecto a abordar, según Lieberman (2007), sería el conocimiento de uno mismo, entendido como el conjunto de habilidades que permiten la autorepresentación, permitiendo la diferenciación del resto de personas, así como la capacidad de regular el propio comportamiento. En esta línea, el bienestar y la salud mental se relacionan con el desarrollo del funcionamiento mental idóneo (GARCÍA-ALANDETE, 2014; VÁZQUEZ et al., 2009).

Los resultados del GHQ-12 permiten concluir que no hay diferencias entre ambos grupos a términos de salud mental, tal y como se esperaba, y en contraposición a las investigaciones que afirman que el alumnado con AACC presenta un mayor grado de vulnerabilidad respecto a sus pares (BAILEY, 2011; RAMIRO et al., 2016). No obstante, ambos grupos superan la puntuación de corte establecida por los autores, indicando que la muestra podría presentar síntomas asociados a posibles trastornos emocionales, entre ellos, depresión y/o ansiedad.

Sin embargo, no se apreciaron diferencias significativas en bienestar, medidas a través del Kidscreen-27, a excepción de la dimensión de "Apoyo Social y Pares", que refleja un menor ajuste del alumnado con AACC, reportando la percepción de un menor apoyo recibido y/o una peor calidad de la interacción con sus iguales por parte de este alumnado con AACC.

Un segundo aspecto, propuesto por Lieberman (2007), sería la comprensión de los demás, entendido como aquellas habilidades que nos permiten predecir, conocer, comprender, e incluso, llegar a sentir o inferir los estados mentales de los demás. Los resultados obtenidos en empatía, a través del Interpersonal Reactivity Index (IRI) (DAVIS, 1980) permiten concluir que no hay diferencias significativas en esta variable entre el alumnado con AACC y la muestra comunitaria, tanto en su vertiente cognitiva como afectiva.

Sin embargo, se aprecian diferencias significativas en ToM, a favor del alumnado de AACC en las dimensiones "Historias mentales" e "Historias físicas", evidenciándose, respectivamente, en primer lugar, una mayor capacidad del alumnado con AACC para inferir estados mentales, mostrando aptitudes mentalistas avanzadas superiores y, en segundo lugar, una mayor capacidad mnésica y de comprensión de textos. Cabe destacar que en la prueba de 
Historias Extrañas de Happé, las "Historias físicas" y las "Historias desvinculadas" actúan como un control, dado que conllevan razonamientos sobre estados físicos y memorísticos, respectivamente, lo que resulta de especial interés en grupos poblacionales con un rendimiento cognitivo superior y problemática en ToM (AGUILAR et al., 2014).

El tercer aspecto, propuesto por Lieberman (2007), sería la interacción producida entre el mundo interno y el externo, entendido como aquellas habilidades que permiten una interacción social satisfactoria. Los resultados en esta variable, medida a través de Habilidades sociales de Goldstein (1980) indican que el alumnado con AACC percibe un desempeño significativamente menor de las dimensiones: "Primeras habilidades sociales" y "Habilidades relacionadas con los sentimientos", lo produciría dificultades para establecer un primer contacto con sus pares y dificultades para expresar, comprender y conocer los sentimientos propios y ajenos (GOLDSTEIN, 1980).

Los resultados obtenidos en la presente investigación se ajustan a los descubiertos por Leiva (2007), que pese a observar un mayor desempeño mentalista en el alumnado con AACC, concluyó que su desarrollo socioemocional y de adaptación social no da soporte a unas mayores competencias socioemocionales y de adaptación social. Es preciso señalar, que pese a percibir un desempeño social menor del alumnado con AACC en determinadas escalas del Kidscreen-27 y de la prueba de Habilidades sociales de Goldstein, lo que cuestiona la capacidad de ajuste social con sus compañeros y exige una mayor investigación en este terreno.

A modo de síntesis, la relación entre la $\mathrm{CS}$ y las $\mathrm{AACC}$ se presenta aún como un área de estudio que necesita más investigación, ya que, aunque la CS se plantee como un constructo que pretende dar explicación a los procesos que subyacen al entendimiento las relaciones con el entorno, partiendo del yo y de las relaciones interpersonales, se encuentra mediatizada tanto por variables de naturaleza tanto cognitivas como afectivas (LIEBERMAN, 2007). Los resultados obtenidos señalan que el alumnado de altas capacidades, en comparación con la muestra comunitaria, no muestra diferencias en el conocimiento de uno mismo, un peor desempeño en algunos aspectos de la interacción producida entre el mundo interno y el externo, mientras que se presenta una mayor habilidad en ToM, que claramente tiene un componente cognitivo, aspecto en el que este alumnado da sus mejores resultados.

Una de las principales limitaciones del presente estudio es que no ha tomado en cuenta otro de los aspectos de la CS, esto es, variables tales el autocontrol, el autoconcepto y las funciones ejecutivas. En futuras investigaciones, sería deseable incluir dichas variables, lo que permitiría conocer, en mayor grado, la influencia de la inteligencia en el constructo de la CS. 
En futuras investigacionessería interesante replicar el estudio, logrando un mayor tamaño muestral o realizando una validación cruzada con otra muestra similar o perteneciente a otra zona geográfica.

AGRADECIMENTOS: A todos los adolescentes e instituciones participantes en el estudio.

\section{REFERENCIAS}

ADOLPHS, R. Social cognition and the human brain. Trends in Cognitive Sciences, v. 3, n. 12, p. 469-479, 1999. DOI: https://doi.org/10.1016/S1364-6613(99)01399-6

ADOLPHS, R. The neurobiology of social cognition. Current opinion in neurobiology, v. 11, n. 2, p. 231-239, 2001.DOI: https://doi.org/10.1016/S0959-4388(00)00202-6

AGUILAR, M. J. et al. Aportes empíricos a la validación y adaptación al español de la tarea mentalista de Historias Extrañas. Revista Argentina de Ciencias del Comportamiento, v. 6, n. 2, p. 1-10, 2014. Disponible en: http://www.redalyc.org/articulo.oa?id=333431788001. Acceso: 10 jun. 2021.

AGUILAR, M. J. et al. La teoría de la mente como proceso mediador del funcionamiento social. Evaluación en el Síndrome de Turner. Revista Argentina de Ciencias del Comportamiento, v. 8, n. 3, p. 40-47, 2016.

BAILEY, C. L. An examination of the relationships between ego development, dabrowski's theory of positive disintegration, and the behavioral characteristics of gifted adolescents.

Gifted Child Quarterly, v. 55, n. 3, p. 208-222, 2011. DOI:

https://doi.org/10.1177/0016986211412180

BANDURA, A.; MCCLELLAND, D. C. Social learning theory. New Jersey: Englewood cliffs Prentice Hall, 1977. v. 1.

BEER, J. S.; OCHSNER, K. N. Social cognition: A multi level analysis. Brain Research, v. 1079, n. 1, p. 98-105, 2006. DOI: https://doi.org/10.1016/j.brainres.2006.01.002

BORGES, A.; HERNÁNDEZ-JORGE, C. La superdotación intelectual: algo más que un privilegio. Acta Científica y Tecnológica, v. 10, p. 28-33, 2006.

BORGES, Á.; HERNÁNDEZ-JORGE, C.; RODRÍGUEZ-NAVEIRAS, E. Evidencias contra el mito de la inadaptación de las personas con altas capacidades intelectuales. Psicothema, v. 23 , n. 3, p. 362-367, 2011.

BORGES, Á.; HERNÁNDEZ-JORGE, C.; RODRÍGUEZ-NAVEIRAS, E. Superdotación y altas capacidades intelectuales, tierra de mitos. RIDPSICLO, v. 1, n. 3, p. 1-11, 2016.

BORLAND, J. H. Myth 2: The Gifted Constitute 3\% to 5\% of the Population. Moreover, Giftedness Equals High IQ, Which Is a Stable Measure of Aptitude. Gifted Child Quarterly,

RPGE- Revista on line de Política e Gestão Educacional, Araraquara, v. 25, n. esp. 4, p. 2045-2066, dic. 2021. e-ISSN: 1519-9029 
v. 53, n. 4, p. 236-238, 2009. DOI: https://doi.org/10.1177/0016986209346825

CATTELL, R. B.; CATTELL, A. K. S.; WEISS, R. H. Factor g-R : test de inteligencia no verbal-revisado. Madrid: TEA, 2017.

COHEN, J. A Coefficient of Agreement for Nominal Scales. Educational and Psychological Measurement, v. 20, n. 1, p. 37-46, 1960.

COMES, G. et al. La evaluación psicopedagógica del alumnado con altas capacidades intelectuales. Revista de Educación Inclusiva, v. 1, n. 1, p. 103-117, 2008.

CONDOR, S.; ANTAKI, C. Cognición social y discurso. In: DIJK, T. (Ed.). El discurso como estructura y proceso. Barcelona: Gedisa, 2000. p. 453-489.

CRONBACH, L. J. Coefficient alpha and the internal structure of tests. Psychometrika, v. 16, n. 3, p. 297-334, 1951.

DAVIS, M. H. A multidimensional Approach to Individual Differences in Empathy. JSAS Catalog of Selected Documents in Psychology, v. 10, p. 85, 1980.

DAVIS, M. H. Measuring individual differences in empathy: Evidence for a multidimensional approach. Journal of Personality and Social Psychology, v. 44, n. 1, p. 113-126, 1983. DOI: https://doi.org/10.1037/0022-3514.44.1.113

DECETY, J. The neurodevelopment of empathy in humans. Developmental Neuroscience, v. 32, n. 4, p. 257-267, 2010. DOI: https://doi.org/10.1159/000317771

GALDERISI, S. et al. Toward a new definition of mental health. World psychiatry : official journal of the World Psychiatric Association (WPA), v. 14, n. 2, p. 231-233, 2015. DOI: https://doi.org/10.1002/wps.20231

GARCÍA-ALANDETE, J. Psicología positiva, bienestar y calidad de vida. En-claves del pensamiento, v. 8, n. 16, p. 13-29, 2014.

GOLDBERG, D. P.; WILLIAMS, P. User's guide to the General Health Questionnaire. Windsor,UK: NFER-Nelson, 1988.

GOLDSTEIN, A. Lista de chequeo de habilidades Sociales (LCHS). Madrid: Projective Way, 1980.

HAPPÉ, F. G. E. An advanced test of theory of mind: understanding of story characters' thoughts and feelings by able autistic, mentally handicapped, and normal children and adults. Journal of autism and Developmental disorders, v. 24, n. 2, p. 129-154, 1994. DOI: https://dx.doi.org/10.1007/BF02172093

HERNÁNDEZ-LASTIRI, P.; BORGES, Á.; CADENAS, M. Differences in mechanisms of social interaction of gifted children based on peer acceptance. Acción Psicológica, v. 16, n. 1, p. 13-30, 2019. DOI: https://doi.org/10.5944/ap.16.1.22169

HERRANZ, N. Elaboración de un test adaptativo informatizado para la medida de la 
inteligencia general a través de la teoría de respuesta al ítem. 2017. Tesis (Doctoral) Universidad de La Laguna, 2017.

HOGAN, R. Development of an empathy scale. Journal of Consulting and Clinical Psychology, v. 33, n. 3, p. 307-316, 1969. DOI: https://doi.org/10.1037/h0027580

HUMFRESS, H. et al. General and relationship-specific models of social cognition: explaining the overlap and discrepancies. Journal of Child Psychology and Psychiatry, v. 43, n. 7, p. 873-883, 2002. DOI: https://doi.org/10.1111/1469-7610.0013_7

LEIVA, V. L. Competencias mentalistas en niños y adolescentes con altas capacidades cognitivas implicaciones para el desarrollo socioemocional y la adaptación social. 2007. Tesis (Doctoral) - Universidad Autónoma de Madrid, 2007.

LIEBERMAN, M. D. Social cognitive neuroscience: a review of core processes. Annual Review of Psychology, v. 58, n. 1, p. 259-289, 2007. DOI: https://doi.org/10.1146/annurev.psych.58.110405.085654

LÓPEZ, L. Los orígenes del concepto de inteligencia I: un recorrido epistemológico desde el mundo clásico hasta el Siglo de las luces. Revista Galego-Portuguesa de Psicoloxía e Educación, v. 21, p. 35-47, 2013.

LÓPEZ, V.; SOTILLO, M. Giftedness and social adjustment: evidence supporting the resilience approach in Spanish-speaking children and adolescents. High Ability Studies, v. 20, n. 1, p. 39-53, 2009. DOI: https://doi.org/10.1080/13598130902860739

MARTIN, L. T.; BURNS, R. M.; SCHONLAU, M. Mental Disorders Among Gifted and Nongifted Youth: A Selected Review of the Epidemiologic Literature. Gifted Child Quarterly, v. 54, n. 1, p. 31-41, 2010. DOI: https://doi.org/10.1177/0016986209352684

MESTRE, V.; FRÍAS, M. D.; SAMPER, P. La medida de la empatía: análisis del Interpersonal Reactivity Index. Psicothema, v. 16, n. 2, p. 255-260, 2004.

OLSZEWSKI-KUBILIUS, P.; SUBOTNIK, R. F.; WORRELL, F. C. Antecedent and concurrent psychosocial skills that support high levels of achievement within talent domains. High Ability Studies, v. 26, n. 2, p. 195-210, 2015. DOI:

https://doi.org/10.1080/13598139.2015.1095077

PFEIFFER, S. El Modelo Tripartito sobre la alta capacidad y las mejores prácticas en la evaluación de los más capaces. Revista de Educacion, n. 368, p. 66-95, 2015.

PFEIFFER, S. Identificación y evaluación del alumnado con altas capacidades: una guía práctica. La Rioja: Universidad Internacional de La Rioja, 2017.

PREMACK, D.; WOODRUFF, G. Does the chimpanzee have a theory of mind? Behavioral and Brain Sciences, v. 1, n. 4, p. 515-526, 1978. DOI:

https://doi.org/10.1017/S0140525X00076512

RAMIRO, P. et al. Bienestar psicológico en personas con alta capacidad intelectual. European Journal of Education and Psychology, v. 9, n. 2, p. 72-78, 2016. DOI: 
https://doi.org/10.1016/j.ejeps.2015.12.001

RAVENS-SIEBERER, U. et al. The KIDSCREEN-52 quality of life measure for children and adolescents: psychometric results from a cross-cultural survey in 13 european countries. Value in Health, v. 11, n. 4, p. 645-658, 2008. DOI: https://dx.doi.org/10.1111/j.15244733.2007.00291.x

RENZULLI, J. S. A practical system for identifying gifted and talented students. Early Child Development and Care, v. 63, n. 1, p. 9-18, 1990. DOI:

https://dx.doi.org/10.4324/9780429469008-2

RODRÍGUEZ-NAVEIRAS, E. et al. Differences in working memory between gifted or talented students and community samples: A meta-analysis. Psicothema, v. 31, n. 3, p. 255262, 2019. DOI: https://doi.org/10.7334/psicothema2019.18

ROEPER, A. How the gifted cope with their emotions. Roeper Review, v. 5, n. 2, p. 21-24, 1982. DOI: https://doi.org/10.1080/02783198209552672

RYAN, R. M.; DECI, E. L. On happiness and human potentials: a review of research on hedonic and eudaimonic well-being. Annual Review of Psychology, v. 52, n. 1, p. 141-166, 2001. DOI: https://doi.org/10.1146/annurev.psych.52.1.141

SÁNCHEZ-LÓPEZ, M. P.; DRESCH, V. The 12-Item General Health Questionnaire (GHQ12): reliability, external validity and factor structure in the Spanish population. Psicothema, v. 20, n. 4, p. 839-43, 2008. Disponible en http://www.redalyc.org/articulo.oa?id=72720450. Acceso: 10 jun. 2021.

SASTRE-RIBA, S. Niños con altas capacidades y su funcionamiento cognitivo diferencial. Revista de Neurología, v. 46, n. S01, p. S11, 2008. DOI: http://dx.doi.org/10.33588/rn.46S01.2008008

SASTRE-RIBA, S. Intervención psicoeducativa en la alta capacidad: funcionamiento intelectual y enriquecimiento extracurricular. Revista de Neurología, v. 58, p. 89-98, 2014. DOI: https://doi.org/10.33588/rn.58S01.2014030

STOTLAND, E. Exploratory Investigations of Empathy. In: BERKOWITZ, L. (Ed.). Advances in experimental social psychology. New York: Academic Press, 1969. v. 4, p. 271-314. DOI: https://doi.org/10.1016/S0065-2601(08)60080-5

TOMAS, A. Conducta tipo A y habilidades sociales en estudiantes del primer al quinto año de psicología de una universidad nacional de Lima. 1995. Tesis (Licenciatura) Universidad Federico Villarreal, 1995.

VALADEZ, M. D. et al. Emotions and reactions to the confinement by COVID-19 of children and adolescents with high abilities and community samples: a mixed methods research study. Frontiers in Psychology, v. 11, p. 2881, 2020. DOI: https://doi.org/10.3389/fpsyg.2020.585587

VALADEZ, M. D.; BETANCOURT, J.; ZAVALA, M. A. Alumnos superdotados y talentosos: Identificación, evaluacion e intervencion. Una perspectiva para docentes. México: 
El Manual Moderno, 2006.

VÁZQUEZ, C. et al. Bienestar psicológico y salud: Aportaciones desde la Psicología Positiva. Anuario de Psicología Clínica y de la Salud, v. 5, n. 1, p. 15-28, 2009.

WIMMER, H. Beliefs about beliefs: representation and constraining function of wrong beliefs in young children's understanding of deception. Cognition, v. 13, n. 1, p. 103-128, 1983. DOI: https://doi.org/10.1111/j.1467-8624.2009.01319.x

\section{Cómo referenciar este artículo}

QUINTERO RODRÍGUEZ, R.; AGUIRRE DELGADO, T.; BORGES DEL ROSAL, M. Á. Altas capacidades y cognición social. Revista on line de Política e Gestão Educacional, Araraquara, v. 25, n. esp. 4, p. 2045-2066, dic. 2021. e-ISSN:1519-9029. DOI: https://doi.org/10.22633/rpge.v25iesp.4.15939

Enviado: 20/08/2021

Revisiones necesarias: $12 / 10 / 2021$

Aprobado: 15/11/2021

Publicado: 08/12/2021 